\title{
Nanofabrication and Growth Processes with Ultra-High Resolution Electron Beam Induced Deposition on Thin Films
}

\author{
W.F. van Dorp*, P. A. Crozier**, C.W. Hagen* and P. Kruit* \\ * Delft University of Technology, Lorentzweg 1, 2628 CJ Delft, The Netherlands \\ **Center for Solid State Science, Arizona State University, Tempe, AZ 85287-1704, USA
}

Developing techniques for controlled fabrication of nanostructures is a topic of intense research and is critical to exploit the full potential of nanotechnology. An enormous variety of approaches utilizing self-assembly are being developed but most of these approaches result in either randomly positioned features of ill-defined size (e.g. island formation through Stranski-Krastanov type mechanism) or uniform particles and periodic arrays using highly controlled solution chemistry. Electron beam induced deposition (EBID) can be used to deposition and precisely position arbitrary shaped nanostructure onto substrates. Such an approach may provide an effective method for fabrication of masks and templates for nanofabrication projects. Several groups have noticed that linewidths of several nanometers are possible with this technique when high energy focused electron beams are employed [1,2,3]. However, many aspects of the deposition and fabrication processes are not well understood at the nanometer level. Here we present work on W nanostructures synthesized using the EBID approach with line widths approaching $1 \mathrm{~nm}$.

Nanostructure synthesis and characterization was conducted in a Tecnai environmental transmission electron microscope (ETEM) allowing the entire growth process to be observed and controlled in situ and in real time. The microscope is equipped with a field emission electron source operating at $200 \mathrm{kV}$ and has a nominal beam spot size of $\sim 0.2 \mathrm{~nm}$ with a current of approximately $20 \mathrm{pA}$. The microscope is fitted with an environmental cell and essentially operates as a cold-wall CVD reactor. Reactive gases are admitted into a sample chamber at low pressures and are allowed to react on resistively heated substrates. The $\mathrm{W}$ nanostructures were deposited onto a $\mathrm{Si}_{3} \mathrm{~N}_{4}$ membrane utilizing $\mathrm{W}(\mathrm{CO})_{6}$ precursor. In a typical experiment, the precursor flux was admitted directly into the chamber via a precision leak valve, the electron beam was focused to a point on the substrate surface causing an immediate reaction that produced highly localized growth of W.

Figure 1 shows a Z-contrast STEM image and intensity linescan from part of a periodic array of $\mathrm{W}$ dots grown at $100^{\circ} \mathrm{C}$ and precursor pressure of $\sim 10^{-3}$ Torr. The full width half maxima of these dots is $1.2-1.5 \mathrm{~nm}$ proving that linewidths approaching $1 \mathrm{~nm}$ can be achieved with this method. Figure 2 shows another array of dots used to investigate the deposition process in more detail. Two sets of aligned dots are deposited on each side of the thin film when EBID is performed with high energy electrons. Tilting the sample reveals the two sets of $\mathrm{W}$ dots (Figure $2 \mathrm{~b}$ ) on the entrance and exit side of the film. The dot intensity alternates along the columns with the bright dots corresponding to the exit surface. Image intensity analysis shows that there is almost twice as much tungsten deposited on the exit surface compared to the entrance surface. Thus there is a strong anisotropy in the flux of those secondary electrons that are most efficient at precursor dissociation. This is a consequence of the momentum transfer direction of the primary fast electron scattering event which is correlated with the momentum distribution of the excited continuum electron. The secondary electrons responsible for precursor dissociation either come from this initial excitation event or are related to this event mainly through plural scattering (since these events preserve the directionality of the primary momentum transfer). This result agrees with recent Monte Carlo simulations [4]. 


\section{References}

[1] Y. N. Silvis-Cividjian et al, Appl. Phys. Lett. 82, 3514 (2003)

[2] K. Mitsuishi et al, Appl. Phys. Lett., 83, 2064 (2003).

[3] P.A. Crozier et al, Appl. Phys. Lett., 84, 3441 (2004)..

[4] Z.Q. Liu et al, J. Appl. Phys. 96, p3983 (2004) by?).
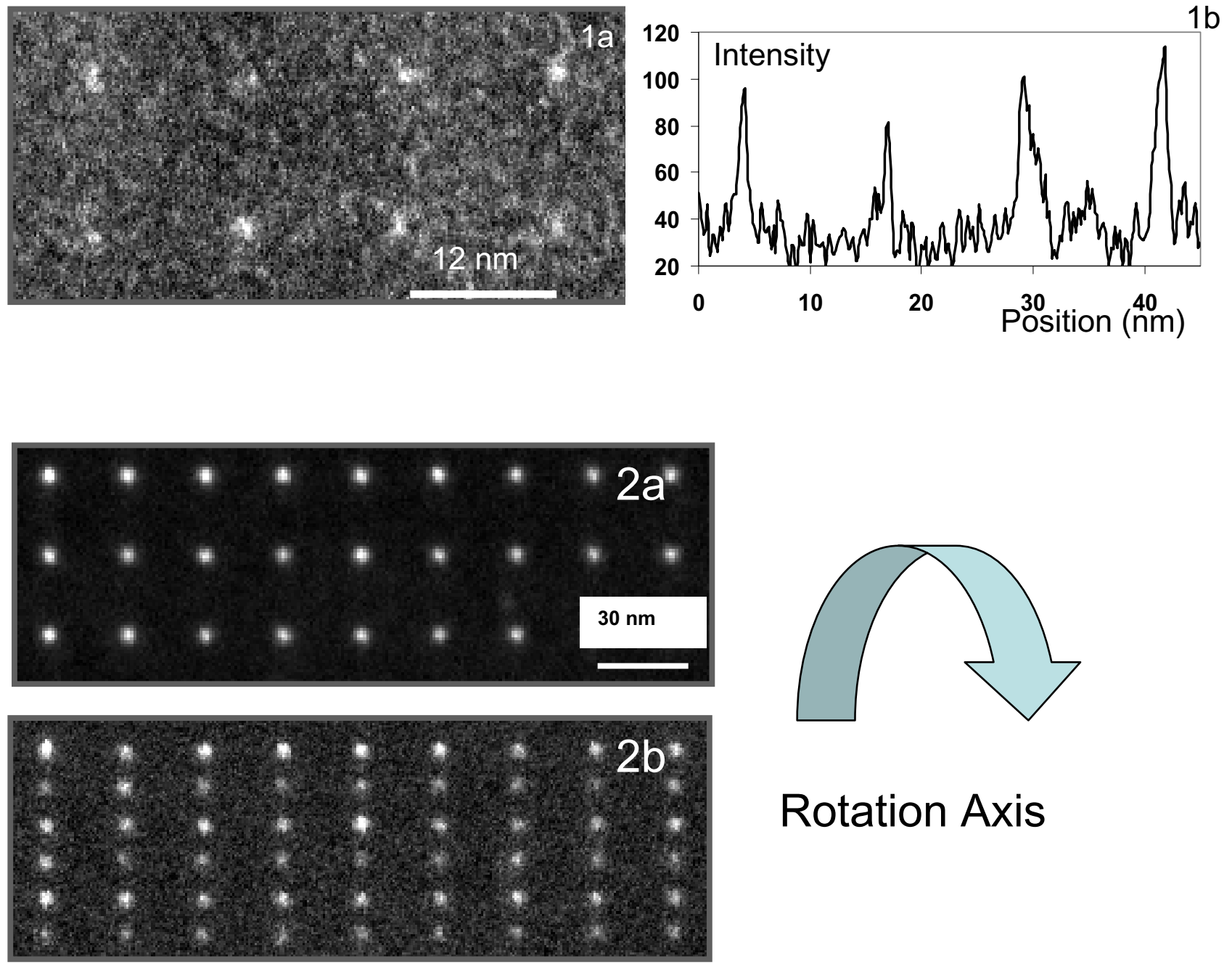

\section{Rotation Axis}

Figure 1: Z-contrast image (a) and associated line scan (b) show W dots with FWHM $\sim 1 \mathrm{~nm}$.

Figure 2: a) Z-contrast image of a periodic array of $3 \mathrm{~nm}$ dots of $\mathrm{W}$ deposited in situ on $\mathrm{Si}_{3} \mathrm{~N}_{4}$. b) image from same area after tilting through $25^{\circ}$ and removal of substrate background. Rows 1, 3 and 5 correspond to exit surface, rows 2, 4 and 6 correspond to entrance surface. 